\title{
De dispositivo de apoio para serviço estruturante: elementos de acoplamento de um AVA ao seu ecossistema
}

\author{
Henri F. Eberspacher ${ }^{1}$, Claude R. Tarrit ${ }^{2}$, Luiz A. de P. Lima Jr ${ }^{1}$ \\ ${ }^{1}$ PPGIa - Programa de Pós-Graduação em Informática \\ PUCPR - Pontifícia Universidade Católica do Paraná - Curitiba - PR \\ 2 Trigone-CIREL - Sciences de l'Éducation \\ Université des Sciences et Technologies de Lille I - Lille - France \\ \{henri, laplima\}@ppgia.pucpr.br, claudetarrit@gmail.com
}

\begin{abstract}
This paper describes a characterization of the coupling between an LMS and the academic environment in which it is inserted. This approach of mutual influence relationship between the various users of the services is built with an approximation of the LMS usage with the systemic organization of influence or development contexts proposed by Bronfenbrenner, thus exemplifying the strong engagement and reciprocity between individuals and the virtual environment and the degree to which them are dependent upon each other.
\end{abstract}

Resumo. Este artigo apresenta uma caracterização do acoplamento de um AVA ao ecossistema universitário no qual está inserido. Esta estruturação das relações de influência mútua entre os diversos usuários dos serviços é elaborada através de uma aproximação dos espaços de uso do AVA com a organização sistêmica dos ambientes de influência ou contextos de desenvolvimento proposta por Bronfenbrenner, permitindo assim exemplificar o forte acoplamento e a reciprocidade entre indivíduos e o ambiente.

\section{Introdução}

A adoção massiva de Ambientes Virtuais de Aprendizagem por Instituições de Ensino Superior, iniciada no Brasil no final da década de noventa, com forte consolidação de experiências nacionais de desenvolvimento e implantação em larga escala durante os anos que se seguiram, está passando por um processo de mercantilização e ruptura das experiências acadêmicas com frequente adoção de plataformas associadas a soluções de fornecedores internacionais (ambiente comerciais ou open-source). Nos EUA, cujo mercado doméstico já em 1993 tinha mais de 90\% das universidades adotando massivamente um LMS [eCampusNews 2016], este movimento de troca de plataforma vem se intensificando, sendo que em torno de $50 \%$ das instituições está em fase de avaliação para substituição de seu atual ambiente [Hill 2016], seja o atual desenvolvido in-loco (com os problemas inerentes de manutenção e de atualização tecnológica no longo prazo) ou uma solução de mercado (que não mais esteja atendendo aos requisitos institucionais). 
O processo de virtualização aplicada ao contexto educacional superior no país tem início em 1997, sobretudo com o desenvolvimento e implantação dos ambientes pioneiros TelEduc [Cerceau 1998 e Rocha 2002], desenvolvido no NIED/Unicamp; AulaNet [Lucena 1998], desenvolvido pelo LES/PUC-Rio e durante muito tempo um dos ambientes virtuais de aprendizagem mais usados no país e o AVA Virtus, criado e desenvolvido pela Universidade Federal de Pernambuco. O ambiente Eureka, cujo experiência de desenvolvimento e uso será o espaço de exploração deste artigo, é contemporâneo destas iniciativas. O desenvolvimento inicial do Eureka data de 1998 como sequência aos trabalhos de [Eberspacher et al. 1997], sendo que foi adotado massivamente na PUCPR a partir do biênio 1999/2000 [Eberspacher et al. 1999].

Diversos fatores estão contribuindo para que as instituições procurem por novos ou alternativos AVAs, entre eles:

(i) disponibilidade e qualidade do serviço, representada atualmente por uma solução em cloud de alta disponibilidade (e consequente custo);

(ii) adaptabilidade e personalização das ferramentas às metodologias ativas de aprendizagem (entre elas Flipped Classroom, Blended Learning e Peer Instruction) e elaboração de trilhas de aprendizagem [Ramos et al. 2015] e monitoramento [Tarrit et al. 2010a e 2010b];

(iii) integração do ecossistema móvel representado por requisitos de m-Learning [Silva et al. 2015]; e

(iv) interoperabilidade técnica seguindo padrões, característica presente nos novos ambientes em contraponto com as dificuldade de integração dos AVAs legados com os ERP acadêmicos.

Neste artigo serão discutidos aspectos históricos, metodológicos, quantitativos e qualitativos, a partir da visão da Bioecologia do Desenvolvimento Humano de Urie Bronfenbrenner [Bronfenbrenner 1979 e 1996], respaldados por mais de 15 anos de experiência na adoção massiva do Eureka na PUCPR [Gomes et al. 2003 e 2006] e [Eberspacher et al. 2003] que atualmente também está em fase de customização e treinamento para adoção em larga escala de uma solução LMS padrão de mercado (BlackBoard).

Esta estruturação aplicando os contextos de desenvolvimento de Bronfenbrenner permitirá uma discussão estruturada sobre o forte acoplamento que ocorre entre um AVA e os processos institucionais, as práticas acadêmicas e o conjunto de usuários, fazendo com que a plataforma não seja simplesmente um serviço de apoio tecnológico para adoção de TDICs na educação, mas tornando-a um elemento fundamental de facto, vinculado ao funcionamento do ecossistema universitário. Esta realidade de simbiose e dependência mútua nem sempre é compreendida em sua amplitude, profundidade e complexidade, o que por vezes traz para o universo das TDICs a frequente percepção de software como commodity (contribuindo decisivamente no movimento atual de substituição de plataformas).

\section{A Bioecologia do Desenvolvimento Humano}

Os trabalhos de [Bronfenbrenner 1979] constituíram um paradigma na Pscicologia do Desenvolvimento Humano no final da década de 70. [Garbarino 1992] sintetiza este esforço de pesquisa como um estudo da interação mútua entre dois entes, os indivíduos em constante crescimento, o indivíduo que aprende e o indivíduo que ensina, assim como 
V Congresso Brasileiro de Informática na Educação (CBIE 2016)

Anais do XXVII Simpósio Brasileiro de Informática na Educação (SBIE 2016)

os meios imediatos e distantes no qual este indivíduo está inserido, sendo este processo influenciado pelas relações entre os contextos mais imediatos e os contextos mais distantes no qual tal indivíduo está integrado.

A integração do indivíduo com o ambiente é caracterizada pela reciprocidade. Como esclarece [Yunes 2010], o indivíduo em desenvolvimento se molda, muda e recria o meio no qual se encontra e o ambiente também exerce influência no desenvolvimento da pessoa, sendo estabelecido então um ambiente de mútua interação.

Para a organização sistêmica dos ambientes de influência ou contextos de desenvolvimento, Bronfenbrenner estabelece seis níveis estruturais do mapa ecológico: onto, micro, meso, exo, macro e cronossistemas. Estes ambientes são como estruturas concêntricas que tem entre elas ou nelas relações funcionais de força variáveis.

O ontossistema forma um sistema contendo as caraterísticas intrínsecas, tais como as competências, as posturas, as relações constitutivas e construtivas de um indivíduo.

O microssistema é um padrão de atividades, papéis e relações interpessoais vivenciados pelo indivíduo em desenvolvimento num dado ambiente com características físicas e materiais específicas. Este contexto se materializa em um local no qual a pessoa pode estabelecer interações face a face com outras pessoas, objetos e símbolos.

O mesossistema é constituído das inter-relações entre os contextos em que o indivíduo participa, sendo que as forças do mesossistema são originadas nas interrelações de dois ou mais ambientes em que este participa ativamente, compondo sua rede social. Uma avaliação deste contexto pode evidenciar interconexões ou desconexões entre dois microssistemas de desenvolvimento. Os contextos microssistêmicos que compõem o mesossistema devem se comunicar, ou seja, há a necessidade de transferência de informações de um contexto para outro (de forma unilateral ou bilateral).

O exossistema é formado pelos ambientes nos quais o indivíduo não possui participação ativa, mas que podem afetar o microssistema no qual este está inserido. Neste contexto são importantes a comunicação bidirecional e o desenvolvimento de objetivos comuns baseados em informações confiáveis e equilíbrio de poder, pois as decisões tomadas neste contexto podem afetar fortemente o indivíduo, mesmo que realizada de maneira indireta.

O macrossistema se refere à consistência na forma e conteúdo dos onto, micro, meso exo e cronossistemas envolvidos, ou seja, ele abrange os demais contextos e tem em sua composição conceitos como cultura, valores, crenças e ideologias. Ele consiste de um padrão externo de um determinado contexto social maior.

$\mathrm{O}$ cronossistema se refere à dimensão temporal. Neste proposito, ele apreende os eventos cronológicos que interferem na relação entre os demais contextos de desenvolvimento. Permite não somente entender as ocorrências de eventos no curto e longo prazo, mais também os ciclos de eventos.

Complexas relações se estabelecem entre os níveis onto, micro, meso, exo, macro e cronossistema. Há um jogo constante de influências entre estes níveis, cada esfera deixa suas marcas no dispositivo educacional que envolve o AVA e cada esfera se apropria dele como ferramenta, neste caso, a plataforma é utilizada para resolver os problemas diretamente relacionados com a esfera de influência do nível estrutural utilizando 
V Congresso Brasileiro de Informática na Educação (CBIE 2016)

Anais do XXVII Simpósio Brasileiro de Informática na Educação (SBIE 2016)

negociações constantes. Esses relacionamentos podem gerar efeitos perturbadores ou previsíveis no dispositivo educacional.

\section{O acoplamento do AVA aos contextos de desenvolvimento}

Em uma aproximação entre os ambientes de influência com o acoplamento de um AVA à estrutura universitária, consideramos os contextos de desenvolvimento conforme apresentado na Tabela 1.

Tabela 1. Acoplamento do AVA aos contextos de desenvolvimento

\begin{tabular}{|c|l|l|}
\hline Contexto & \multicolumn{1}{|c|}{ Posicionamento } & \multicolumn{1}{c|}{ Materialização } \\
\hline ontossistema & contexto interno & $\begin{array}{l}\text { definição das necessidades técnicas e de } \\
\text { usabilidade, projeto do sistema, banco de } \\
\text { dados, etc. }\end{array}$ \\
\hline microssistema & contexto direto & $\begin{array}{l}\text { condições espaço-temporais, natureza do } \\
\text { dispositivo, recursos-humanos envolvidos }\end{array}$ \\
\hline mesossistema & contexto mais próximo & $\begin{array}{l}\text { nível institucional próximo, curso que } \\
\text { frequenta, salas virtuais/disciplinas do } \\
\text { curso }\end{array}$ \\
\hline exossistema & contexto intermediário & $\begin{array}{l}\text { politicas institucionais, decisões } \\
\text { estratégicas, elementos obrigatórios da } \\
\text { IES }\end{array}$ \\
\hline macrossistema & $\begin{array}{l}\text { contexto mais afastado do do } \\
\text { ensino/aprendizagem }\end{array}$ & $\begin{array}{l}\text { política educacional nacional, currículos } \\
\text { de referência, práticas legais obrigatórias }\end{array}$ \\
\hline cronossistema & contexto temporal & $\begin{array}{l}\text { período de desenvolvimento, de } \\
\text { integração ou de manutenção, ciclos de } \\
\text { atividades técnicas e pedagógicas ou de } \\
\text { gestão dessas atividades, etc. }\end{array}$ \\
\hline
\end{tabular}

O contexto ecológico do AVA também se manifesta:

- enquanto ontossistema: na modificação das práticas de ensino, de aprendizagem e de gestão; bem como na formação de esquemas de uso e de operação;

- enquanto microssistema: no Ambiente Virtual de Aprendizagem e nos dispositivos vinculados que fazem parte do ambiente imediato do estudante em suas atividades corriqueiras relacionadas ao cumprimento de seu papel como indivíduo ativo em uma sala virtual/disciplina de curso; e no caso do professor como atuante na liberação e na mediação do seu espaço enquanto interlocutor privilegiado junto aos estudantes (ampliação do microssistema da sala de aula no dual virtual);

- na industrialização do messosistema: colocando o AVA a disposição da totalidade da instituição como ferramenta de apoio ao ensino presencial e a distância (criando uma sala para cada disciplina ofertada regularmente no campus de forma automática);

- enquanto exossistema: devido a institucionalização com as infraestruturas (de TI, como bancos de dados/ERP Acadêmico) e a multiplicação dos dispositivos (o AVA como um canivete suíço ou o AVA como um referencial); 
V Congresso Brasileiro de Informática na Educação (CBIE 2016)

Anais do XXVII Simpósio Brasileiro de Informática na Educação (SBIE 2016)

- enquanto macrossistema: devido aos eventuais problemas trabalhistas (professores usando em horários não acordados legalmente, sobrecarga de trabalho para formatação de conteúdo, sobrecarga de comunicação com estudantes etc.) e demais situações legais vinculadas aos estudantes e professores (processos, geração de documentos/comprovantes etc.);

- enquanto cronossistema: representa os tempos, os ciclos de aprendizagens e o próprio processo histórico de desenvolvimento e de apropriação do AVA.

\section{As tensões no contexto do AVA}

A transição ecológica ocorre cada vez que a posição de um indivíduo é modificada no ambiente em decorrência de uma alteração em suas atividades, relacionamento ou papeis desempenhados. Estas modificações alteram a posição ecológica do indivíduo no contexto.

Estas transições geram tensões que no contexto do AVA são materializadas e potencializadas, sendo que os estudantes e os professores são os atores mais diretamente influenciados pelas políticas de uso institucional do AVA, pois as decisões tomadas no exossistema influenciam diretamente seus microssistemas.

A aplicação massiva de um AVA no contexto universitário, como o caso do AVA Eureka, cria um exossistema que molda e influencia o mesossistema de forma determinante, pois decisões institucionais criam tensões que aparecem no cotidiano da aplicação do ambiente nos cursos. A reciprocidade também ocorre, o uso intensivo do AVA pela totalidade do corpo discente influencia o nível das decisões estratégicas. Este regime de tensão pode levar a rupturas, dependendo da força da influência exercida.

Políticas institucionais, como roteirização obrigatória dos TDEs (Trabalho Discente Efetivo) segundo padrão definido, acompanhamento individualizado da execução dos TDEs por equipe pedagógica de apoio, acesso dos coordenadores de curso a todas as salas virtuais de seus professores, entre outros; possibilitam o acompanhamento e fiscalização da atividade docente, sendo este uma influência direta na atividade fim do professor que modifica sua prática e vice-versa, influenciando o modelo de gestão e acompanhamento. Esta são tensões geradas nas inter-relações entre os contextos de participação ativa do indivíduo, influenciando seu mesossistema. Os ciclos nas atividades de gestão, docente e discente influenciam seu cronossistema.

Para evidenciar as tensões, usamos uma representação inspirada da Teoria da Atividade tal como abordada por [Engeström 1987] que permite visualizar os elementos que incidem sobre estas e entre o indivíduo, o AVA e os contextos. Foram escolhidos elementos definidos em função das atividades vinculadas a inserção do AVA no contexto universitário e dos relacionamentos sistêmicos. Neste artigo consideraremos o pólo denominado Influência, que se apoia justamente na bioecologia de Bronfenbrenner, tal qual representado na Figura 1 [Tarrit 2015].

O pólo Influência permite contextualizar eventos influenciadores remarcáveis na bioecologia e classificar os efeitos de suas relações interpessoais e inter-contextuais. $\mathrm{Na}$ ilustração proposta este pólo é posicionado sobre o Triângulo de Engeström adaptado com os contextos de desenvolvimento. O evento influenciador é considerado quando uma modificação observável ocorre em um contexto como consequência de uma ruptura ou alteração em um outro contexto que o influencia, gerando portanto novas tensões. 
V Congresso Brasileiro de Informática na Educação (CBIE 2016)

Anais do XXVII Simpósio Brasileiro de Informática na Educação (SBIE 2016)



Figura 1. Representação do Pólo Influência [Tarrit 2015], ilustrando um possível evento de influência que gera tensões que se propagam

As transições ecológicas são representadas na Figura 1 por setas espessas e apresentam uma mudança de nível de influência. Cada transição ecológica possui um elemento de acompanhamento que define o vínculo ecológico. A flecha reflexiva (pontilhada), é uma transição ecológica que tem como origem um evento de influência do mesmo contexto ecológico.

No exemplo de propagação evidenciado (setas) na Figura 1 (caso particular), os níveis de influência ilustrados são definidos da seguinte forma:

- Contexto de influência 1: representa uma origem sistêmica secundária, ele tem caráter contextual fraco, pois sua influência (micro sobre meso) não é suficientemente forte para modificar o contexto ecológico geral, porém gera tensões na relação ecológica entre ambos os contextos.

- Contexto de influência 2: representa uma origem sistêmica primária, ele tem um caráter dominante ou requisito forte, pois sua influência (meso sobre meso ou onto sobre exo, no caso do exemplo) são suficientemente fortes para modificar o contexto ecológico geral.

Os estudantes também têm suas tensões exacerbadas em seu microssistema potencializadas pelo ambiente virtual, como exemplificado na Figura 2. 
V Congresso Brasileiro de Informática na Educação (CBIE 2016)

Anais do XXVII Simpósio Brasileiro de Informática na Educação (SBIE 2016)



Figura 2. Tensões do estudante [Choquet 2008]

\section{A influência ecológica no roteiro pedagógico}

A centralidade do professor no dispositivo educacional e na articulação do microssistema virtual no AVA é evidenciada na questão do planejamento pedagógico, no qual determina os objetivos, delimitando temas e recursos, instigando mediante comunicação e planificando o andamento das aulas e de suas atividades (organiza, produz e monitora os recursos do AVA para seus microssistemas).

A Figura 3 apresenta a influência da metodologia proposta (ou imposta) pelo exossistema da IES na concepção do roteiro pedagógico sobre o dispositivo necessário às tarefas de acompanhamento e concretização dos microssistemas do professor. São representadas as 10 dimensões descritas por [Class 2001] que se apoia na escala de [Reeves 1998] e nos modelos de ensino de [Joyce 2000], que divide em quatro famílias os modelos de ensino: socialização, tratamento da informação, individualidade e sistemas behavioristas .

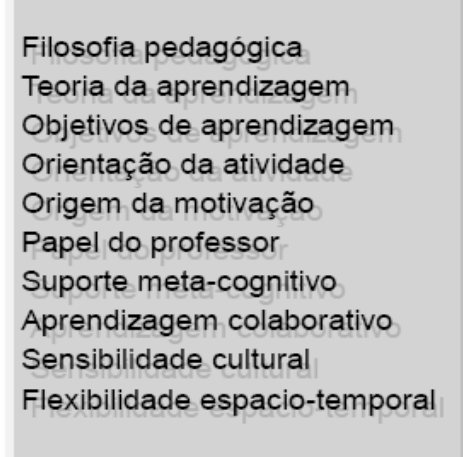

Filosofia pedagógica Teoria da aprendizagem

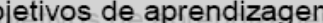
Flexibilidade espacio-temporal Sensibilidade cultural

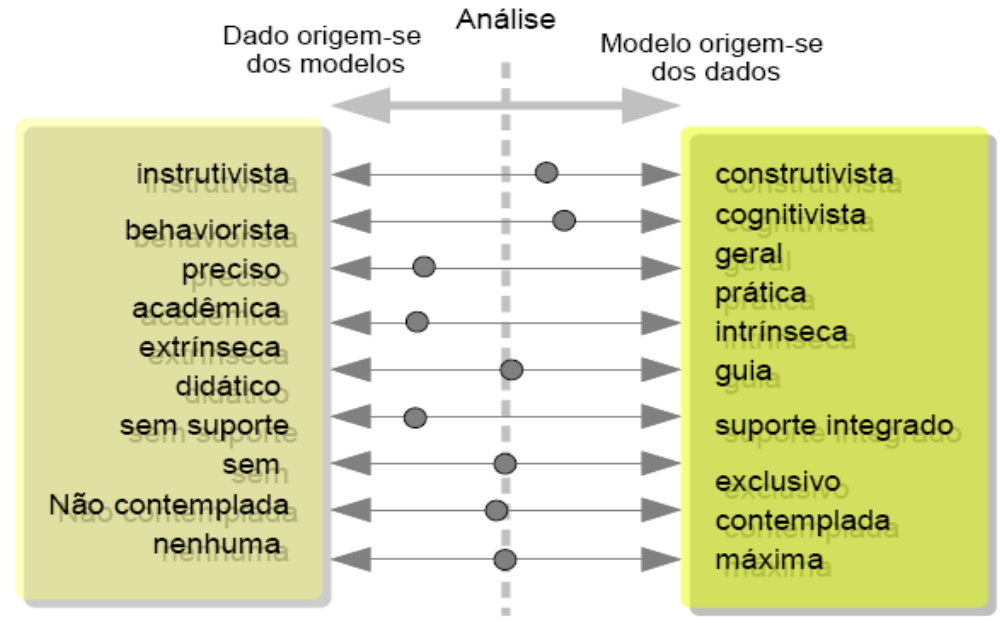

Figura 3. Influência da metodologia na trilha de aprendizagem [Reeves 1998], [Class 2001] e [Tarrit 2015]

Quanto mais as características da trilha de aprendizagem tendem à esquerda da escala, mais o acompanhamento tem uma forma determinada que se origina das ações provocadas pelas atividades planificadas no roteiro. 
À direta da escala a atividade não é fortemente controlada e a tarefa é individualizada, o que reforça o pressuposto de que a atitude está na mão do estudante para realização da atividade, assim ela escapa a um modelo único de resolução. À direita os dados de acompanhamento originam-se do modelo, à esquerda são os dados que permitem que seja definido o modelo de acompanhamento [Tarrit 2010]. Estas características permitirão a avaliação das interconexões ou desconexões no mesossistema.

\section{Considerações finais}

O mercado atual (1o. semestre de 2016) de ambientes virtuais no mundo, apenas na educação superior, está estimado em 1 bilhão e 750 milhões de dólares, sendo aproximadamente $35 \%$ associado a sistemas comerciais proprietários e $65 \%$ associado a sistemas open-source [LISTedTECH 2016]. Nesta estimativa não estão presentes custos com MOOCs e demais sistemas periféricos, apenas a disponibilização dos AVAs no ensino superior, sem considerar Ensino Fundamental e Médio e os sistemas de treinamento corporativo ou produção de conteúdo.

Neste artigo procuramos fazer uma aproximação da experiência vivenciada na PUCPR no desenvolvimento e uso de seu ambiente virtual proprietário quanta a mútua influência entre o AVA e os diversos segmentos da universidade, usando para tal uma estruturação dos atores institucionais segundo a organização sistêmica dos ambientes de influência proposta por Bronfenbrenner na Teoria da Bioecologia do Desenvolvimento Humano.

O uso do Eureka historicamente formatou uma série de práticas pedagógicas, modificando práticas tradicionais centradas no ensino para professores mediadores centrados na aprendizagem; limitou e estimulou uma série de decisões estratégicas institucionais quanto a oferta de formação a distância e apoio presencial, bem como estimulou grande quantidade de pesquisa aplicada em computação e educação, modelando e influenciando decisivamente o ambiente acadêmico e as relações entre seus atores.

Da mesma forma, os ambientes de influência limitaram, modificaram, adaptaram e fizeram uma (re)leitura do uso da plataforma, em um processo de influência mútua que envolveu mais de 40.000 estudantes e 2.000 professores, muitos dos quais usaram o ambiente Eureka durante todo seu ciclo universitário, e até por conta disso vincularam muito da experiência acadêmica aos acertos e erros da prática e uso do AVA.

Esta influência mútua, no caso da apropriação das ferramentas de um AVA, passa também pelo desvirtuamento de funcionalidades, cuja emprego prático pelos docentes e discentes pode se distanciar bastante da função originalmente concebida, interferindo na percepção do ambiente e de sua aplicação, tanto positiva quanto negativamente, entrando aqui os conflitos no mesossistema.

Na realidade exposta de mercado de LMS mundial e mudanças de plataformas para soluções comerciais, uma nova safra de adaptações e influências ainda estão por se concretizar na realidade nacional, associada a m-Learning, adaptabilidade, acessibilidade e recursos inclusivos, entre outros desafios tanto de cunho tecnológico quanto educacional e metodológico (aprendizagens ativas). 
V Congresso Brasileiro de Informática na Educação (CBIE 2016)

Anais do XXVII Simpósio Brasileiro de Informática na Educação (SBIE 2016)

\section{Referências}

Bronfenbrenner, U. (1979). The ecology of human development. Cambridge, MA: Harvard University Press, 1979.

Bronfenbrenner, U. (1996). A ecologia do desenvolvimento humano: experimentos naturais e planejados. Porto Alegre: Artes Médicas, 1996.

Cerceau, A. D. (1998). Formação a Distância de recursos Humanos para a Informática Educativa. Dissertação (Mestrado em Ciência da Computação) - Universidade Estadual de Campinas, Orientador: Heloisa Vieira da Rocha.

Choquet C., Iksal S., Levene M., Schoonenboom J. (2008). Users' data: trails analysis. In: Technology-Enhanced Learning - Principles and Products. Edition: Nicolas Balacheff, StenLudvigsen, Ton de Jong, ArdLazonder, Sally Barnes, Lydia Montandon, Springer Verlag.

Class, B. (2001). De l'éducation présentielle à l'éducation distancielle : quelques concepts et études de cas. Mémo (CTECFA.

Eberspacher, H. F. e Kaestner, C. A. A. (1997). A Arquitetura de um Sistema de Autoria para Construção de Tutores Inteligentes Hipermídia. In: Simpósio Brasilerio de Informática na Educação - SBIE, 1997, São Jose dos Campos. Anais do SBIE96. São José dos Campos: ITA, 1997. p. 163-179.

Eberspacher, H. F.; Vasconcelos, C. D.; Jamur, J. H.; Eleuterio, M. A. (1999). Eureka: um ambiente de aprendizagem cooperativa baseado na Web para Educação à Distância. In: Simpósio Brasileiro de Informática na Educação - SBIE 1999. Anais do SBIE.

Eberspacher, H. F, Kozak, D.V., Souza, L.C.G. ; Campagnolo, B. (2003). A utilização do Eureka nos cursos de engenharia da PUCPR. In: Gomes, P. V., Matos, E.L.M. (Org.). Uma Experiência de Virtualização Universitária: O Eureka da PUCPR. Curitiba, 2003, p. 17-28.

eCampusNews. (2016). Breaking: Billion-dollar e-learning market about to undergo major shift. Disponível on-line em http://www.ecampusnews.com/curriculum/lmsmarket-cloud/ Publicado em 17 de maio de 2016, acessado em 13 junho de 2016.

Engeström, Y. (1987). An Activity Theoretical Approach to Developmental Research. (Orienta-Konsultit, Éd.). Disponível on-line no Laboratory of Comparative Human Cognition em $\quad$ http://lchc.ucsd.edu/mca/Paper/Engestrom/Learning-byExpanding.pdf, acessado em 13 de junho de 2016.

Gomes, P. V.; Matos, E. (Org.). (2003). Uma Experiencia de Virtualizaçao Universitaria - O Eureka da PUPCR. 1. ed. Curitiba: Editora Champagnat, 2003. 191p.

Gomes, P. V.; Mendes, A. M. C. P. (Org.). (2006). Tecnologia e Inovaçao na Educaçao Universitaria: O MATICE DA PUCPR. 1. ed. Curitiba: Editora Champagnat, 2006. $314 \mathrm{p}$.

Garbarino, J. e Abramowitz, R. H. (1992). Sociocultural risk and opportunity. In: Garbarino, James (ed.). Children and families in the social environment. 2.ed. New York: Aldine de Gruyter, 1992. p. 35-70. 
V Congresso Brasileiro de Informática na Educação (CBIE 2016)

Anais do XXVII Simpósio Brasileiro de Informática na Educação (SBIE 2016)

Hill, P. (2015). State of the US Higher Education LMS Market. Disponível on-line em: http://mfeldstein.com/state-of-the-us-higher-education-lms-market-2015-edition/

Publicado em 14 de outubro de 2015, acessado em 13 junho de 2016.

Joyce, B., Weil, M., Calhoun, E. (2000) Models of teaching, 6th edition, Allyn \& Bacon.

LISTedTECH. (2016). Is the LMS a Billion dollar Market? Disponível on-line em http://listedtech.com/higher-education-lms-market-value/ Publicado em 12 de junho de 2016, acessado em 13 junho de 2016.

Lucena C. J. P., Fuks, H., Molidiu, R., Macedo, L., Santos, N., Laufer, C., Ribeiro, M. B., Fontoura, M. F., Noya, R. C., Crespo, S., Torres, V., Daflon, L. e Lukowiecki, L. (1998). AulaNet - an environment for the development and maintenance of courses on the Web. In: Proceedings of the International Conference on Engineering in Education. Rio de Janeiro, RJ.

Ramos, D. B., Oliveira, E. H. T. de, Ramos, I. M. M., Oliveira, K. M. T. (2015). Trilhas de Aprendizagem em Ambientes Virtuais de Ensino-aprendizagem: Uma Revisão Sistemática da Literatura. In: Simpósio Brasileiro de Informática na Educação - SBIE 2015. Anais do SBIE.

Reeves, T.C., Reeves, P.M. (1998). Effective Dimensions of Interactive Learning on the World Wide Web. in Web-Based Instruction, Englewood Cliffs . N.J.: Educational Technology Publications, 1998.

Rocha, H. V., Otsuka, J. L., Lachi, R. L. e Ferreira, T. B. (2002). Suporte à Avaliação Formativa no Ambiente de Educação à Distância TelEduc. In: VI Congresso Iberoamericano de Informática Educativa, Vigo. Anais do VI Congresso Iberoamericano de Informática Educativa, 2002.

Silva, F., Silva, J. K., Lucena, M., Fomes, A. G. (2015). Requisitos para Integração entre Ambientes de Aprendizado e m-Learning: uma Revisão Sistemática da Literatura. In: Simpósio Brasileiro de Informática na Educação - SBIE 2015. Anais do SBIE.

Tarrit, C. R.; Caron, P-A. (2010). Monitoring students in LMS Eureka - Toward new features using trace analyses. In: Simpósio Brasileiro de Informática na Educação SBIE 2010 - III Workshop de Avaliação e Acompanhamento da Aprendizagem. Anais do SBIE.

Tarrit, C. R., Hilu, L. e Kowalski, R. P. G. (2010). Acompanhamento do processo ensino/aprendizado por meio de ambiente virtual de aprendizagem. In: 16 CIEAD Congresso Internacional ABED de Educação a Distância 2010. Anais do CIEAD.

Tarrit, C. R. (2015) Étude d'un Dispositif Pédagogique Instrumenté Complexe et de ses propriétés. Une approche épistémique et méthodologique, le cas d'une université brésilienne. Tese, Sciences de l'Homme et Société. Univ. Lille Cirel-Trigone.

Yunes, M. A. M. e Juliano, M. C. (2010). Bioecologia do Desenvolvimento Humano e suas Interfaces com Educação Ambiental. In: Cadernos de Educação - UFPel, No. 17. 